Received: 7 August 2018

Accepted: 12 November 2018

Published online: 24 December 2018

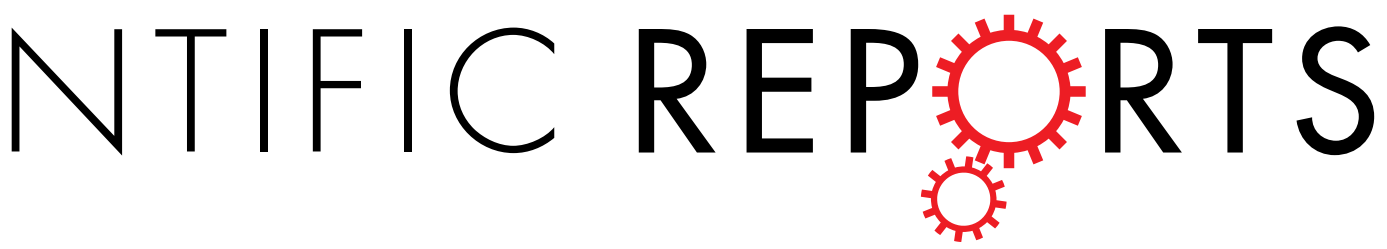

\title{
Adhesive-free adhesion
}

\section{between heat-assisted plasma- treated fluoropolymers (PTFE,} PFA) and plasma-jet-treated polydimethylsiloxane (PDMS) and its application

\author{
Yuji Ohkubo 1 , Katsuyoshi Endo \& Kazuya Yamamura
}

Conventional low-temperature plasma treatment was reported to minimally improve the adhesion property of polytetrafluoroethylene (PTFE), whereas heat-assisted plasma (HAP) treatment significantly improved the same. An unvulcanized rubber was previously used as an adherent for PTFE. This study aimed to achieve strong adhesive-free adhesion between PTFE and vulcanized polydimethylsiloxane (PDMS) rubber. As-received vulcanized PDMS rubber did not adhere to HAPtreated PTFE, and as-received PTFE did not adhere to vulcanized rubber of plasma-jet (PJ) treated PDMS rubber; however, HAP-treated PTFE strongly adhered to vulcanized PJ-treated PDMS rubber, and both PTFE and PDMS exhibited cohesion failure in the T-peel test. The surface chemical compositions of the PTFE and PDMS sides were determined using X-ray photoelectron spectroscopy. The strong PTFE/PDMS adhesion was explained via hydrogen and covalent bond formation (C-O-Si and/or $\mathrm{C}(=\mathrm{O})-\mathrm{O}-\mathrm{Si})$ between hydroxyl $(\mathrm{C}-\mathrm{OH})$ or carboxyl $(\mathrm{C}(=\mathrm{O})-\mathrm{OH})$ groups of the HAP-treated PTFE. This process was also applied to adhesive-free adhesion between a tetrafluoroethyleneperfluoroalkylvinylether copolymer (PFA) and PDMS; subsequently, a translucent PFA/PDMS assembly with strong adhesion was realized together with the PTFE/PDMS assembly. Strong adhesive-free adhesion between fluoropolymers (PTFE, PFA) and vulcanized PDMS rubber without using any adhesives and graft polymer was successfully realized upon plasma treatment of both the fluoropolymer and PDMS sides. Additionally, a PDMS sheet, which was PJ-treated on both sides, was applied to strongly adhere fluoropolymers (PTFE, PFA) to materials such as metal and glass. PJ-treated PDMS was used as an intermediate layer rather than a strong adhesive, achieving PTFE/PDMS/metal and PTFE/PDMS/glass assemblies. The PTFE/PDMS, PDMS/metal, and PDMS/glass adhesion strengths exceeded $2 \mathrm{~N} / \mathrm{mm}$.

Polytetrafluoroethylene (PTFE) comprises only $\mathrm{CF}_{2}$ chains and a typical fluoropolymer. While this fluoropolymer offers several advantages, such as good water and oil repellency, high chemical resistance, weather resistance, and good sliding properties, it does not readily adhere to other types of materials because of its low surface energy and weak boundary layer ${ }^{1,2}$. To overcome its extremely poor adhesion properties, chemical etching using corrosive solutions containing sodium has long been utilized ${ }^{3-5}$. However, chemical etching has several disadvantages, including malodour, toxicity to humans, a high environmental load, and PTFE surface coloration. Therefore, an alternative method that does not require corrosive solutions has long been needed. Specifically, dry processes such as ion irradiation and plasma treatment are potentially suitable alternatives to chemical etching because these present almost no danger to humans together with a low environmental load. Ion irradiation is performed 
under low pressure such as $1.3 \times 10^{-2} \mathrm{~Pa}$. However, plasma treatment can be performed under a wide range of pressures from low to atmospheric pressure, e.g., $1.0 \times 10^{5} \mathrm{~Pa}$; thus, plasma treatment has possibility of having no vacuum evacuation system. This pressure difference indicates that plasma treatment is potentially more suitable than ion irradiation for practical purposes, especially large area continuous processing. However, conventional plasma treatment results in minimal improvement in the adhesion properties of PTFE. Although some reports described on indirect adhesion between PTFE and other types of materials using adhesives ${ }^{6,7}$ and/or graft polymers $^{8-11}$, hardly any reports mentioned on adhesive-free adhesion without graft polymerization. We considered the parameters for the plasma treatment conditions such as pressure, plasma treatment time, and polymer surface temperature. Finally, we developed heat-assisted plasma (HAP) treatment and realized strong adhesive-free adhesion between PTFE and unvulcanized rubber without graft polymerization ${ }^{12}$. On analyzing the PTFE surface, it was concluded that HAP was central to both introducing oxygen-containing functional groups $(\mathrm{O}-\mathrm{C}=\mathrm{O}, \mathrm{C}=\mathrm{O}$, $\mathrm{C}-\mathrm{O}$ ) on the PTFE surface and increasing the surface hardness through $\mathrm{C}-\mathrm{C}$ crosslinking formation and etching during plasma treatment to improve the adhesion properties of PTFE $^{13}$. Additionally, several types of unvulcanized rubber containing different rubber compounding agents, such as crosslinking and reinforcing agents, were prepared; the unvulcanized rubber was then adhered to HAP-treated PTFE to determine which rubber compounding agent was the most effective in improving the adhesion strength. Consequently, it was found that hydrophilic $\mathrm{SiO}_{2}$ powder, having a silanol group $(\mathrm{Si}-\mathrm{OH})$ that functions as a reinforcing agent, contributed to the high adhesion strength between HAP-treated PTFE and unvulcanized rubber ${ }^{14}$. In previous studies ${ }^{12-14}$, unvulcanized rubbers were employed as adherents. In the present study, we focus on vulcanized rubber as an adherent to PTFE. We selected vulcanized polydimethylsiloxane (PDMS) as a vulcanized rubber. It is reported that when a PDMS surface is modified by plasma, corona discharge, and ultraviolet (UV) treatments, then silanol groups $(\mathrm{Si}-\mathrm{OH})$ are readily generated ${ }^{15-17}$. Additionally, PDMS is widely used in the medical industry, especially as a material for microfluid chips ${ }^{18-21}$. Adhesive bonding between PDMS surfaces and adhesive-free adhesion between PDMS surfaces have already been established, and related techniques are reported in many research articles ${ }^{22-24}$. Moreover, both adhesive bonding and adhesive-free adhesion between PDMS and other types of materials (resins such as PMMA, PP, and $\mathrm{PE}^{25-27}$; metals such as $\mathrm{Cu}, \mathrm{Fe}, \mathrm{Pt}, \mathrm{Au}$, and $\mathrm{Al}^{27,28}$; and glass ${ }^{28-30}$ ) were also reported. However, these articles rarely reported on adhesion between PDMS and fluoropolymers, or the adhesion strength between PDMS and PTFE was too low for practical use ${ }^{27,31,32}$. In medical and food industries, adhesives are unlikely to be employ because they are regarded as contaminations. Thus, adhesive-free adhesion is essential in the medical and food industries. Adhesive-free adhesion between PDMS and PTFE using tetrapodal ZnO fillers were reported and the adhesion strength increased upon addition of the fillers, but the maximum adhesion strength was below $0.3 \mathrm{~N} / \mathrm{mm}^{33}$. Thus, the effect of shape of fillers was not enough to strongly adhere PDMS to fluoropolymers. In this study, we aim to achieve strong adhesive-free adhesion based on chemical interaction between vulcanized PDMS and fluoropolymers containing PTFE.

\section{Results}

Surface chemical composition analysis of plasma-treated PDMS and plasma-treated PTFE using X-ray photoelectron spectroscopy (XPS). Figure 1a-1c shows the XPS spectra of the PDMS samples before and after plasma-jet (PJ) treatment. The intensity of the peak indexed to $\mathrm{CH}_{3}$ decreased after PJ treatment, resulting in $\mathrm{C}-\mathrm{Si}$ bond scission and desorption of $\mathrm{CH}_{3}$ on the PJ-treated PDMS surface (Fig. 1a). No peaks indexed to $\mathrm{C}-\mathrm{O}(286.5 \mathrm{eV})^{34,35}, \mathrm{C}=\mathrm{O}(288.0 \mathrm{eV})^{34,35}$, or $\mathrm{O}-\mathrm{C}(=\mathrm{O})(289.2 \mathrm{eV})^{34,35}$ were observed for the PJ-treated PDMS surface (Fig. 1a). The intensity of the O1s peak increased and shifted to higher binding energy after PJ treatment, indicating an oxidation reaction on the PJ-treated PDMS surface (Fig. 1b). The intensity of the Si2p peak also increased and shifted to higher binding energy after PJ treatment, also indicating an oxidation reaction on the PJ-treated PDMS surface (Fig. 1c). Furthermore, peak resolution of O1s-XPS and Si2p-XPS spectra of as-received and PJ-treated PDMS samples was conducted. Figure 1d,1e shows peak resolution of the O1s-XPS spectra of the PDMS samples before and after PJ treatment. Peaks 1 and 2 indicate Si-O-Si and Si$\mathrm{OH}$, respectively ${ }^{36,37}$. The as-received PDMS sample had the ratio $\mathrm{Si}-\mathrm{OH}: \mathrm{Si}-\mathrm{O}-\mathrm{Si}=9: 91$. This also indicated that the surface of the as-received PDMS sample used in this study originally contained ca. $10 \% \mathrm{Si}-\mathrm{OH}$. The PJ-treated PDMS sample had the ratio $\mathrm{Si}-\mathrm{OH}: \mathrm{Si}-\mathrm{O}-\mathrm{Si}=13: 87$. These results indicated that $\mathrm{Si}-\mathrm{OH}$ increased after PJ treatment. Figure 1f,1g shows peak resolution of the Si2p-XPS spectra of the PDMS samples before and after PJ treatment. Peaks $1^{\prime}, 2^{\prime}$ and $3^{\prime}$ indicate $\mathrm{Si}^{2+}:-\left[\mathrm{Si}\left(\mathrm{CH}_{3}\right)_{2}-\mathrm{O}-\right]-, \mathrm{Si}^{3+}:-\left[\mathrm{Si}_{(}\left(\mathrm{CH}_{3}\right)(\mathrm{OH})-\mathrm{O}-\right]-$, and $\mathrm{Si}^{4+}:-$ $\left[\mathrm{Si}(\mathrm{OH})_{2}-\mathrm{O}-\right]-$, respectively ${ }^{36,37}$. As-received PDMS had the ratio - $\left[\mathrm{Si}(\mathrm{OH})_{2}-\mathrm{O}-\right]_{-}:-\left[\mathrm{Si}_{(}\left(\mathrm{CH}_{3}\right)(\mathrm{OH})-\mathrm{O}-\right]_{-}:-$ $\left[\mathrm{Si}\left(\mathrm{CH}_{3}\right)_{2}-\mathrm{O}-\right]-=0: 11: 89$. This $\mathrm{Si} 2 \mathrm{p}-\mathrm{XPS}$ result together with the O1s-XPS result also indicated that the surface of the as-received PDMS sample used in this study originally contained ca. 10\% Si-OH. PJ-treated PDMS had the ratio $-\left[\mathrm{Si}(\mathrm{OH})_{2}-\mathrm{O}-\right]-:-\left[\mathrm{Si}\left(\mathrm{CH}_{3}\right)(\mathrm{OH})-\mathrm{O}-\right]-:-\left[\mathrm{Si}\left(\mathrm{CH}_{3}\right)_{2}-\mathrm{O}-\right]-=6: 14: 80$. These results also suggested that $\mathrm{Si}-$ $\mathrm{OH}$ increased after $\mathrm{PJ}$ treatment. Previous articles ${ }^{15,36,37}$ reported $\mathrm{Si}-\mathrm{C}$ scission and $\mathrm{CH}_{3}$ desorption upon plasma treatment, followed by formation of $\mathrm{Si}-\mathrm{O}$ and/or $\mathrm{Si}-\mathrm{OH}$ upon reaction between Si radicals and oxygen atoms or $\mathrm{OH}^{-}$ions in plasma. These reports are consistent with our study.

Figure 2a,2b shows the XPS spectra of the PTFE samples before and after HAP treatment. The as-received PTFE sample exhibited only a peak indexed to $\mathrm{CF}_{2}$ at ca. $292 \mathrm{eV}$, as shown in Fig. 2a. However, the HAP-treated PTFE sample had peaks indexed to not only fluorine-containing functional groups $\left(\mathrm{CF}_{3}, \mathrm{CF}_{2}, \mathrm{C}-\mathrm{F}\right)^{13,34,35,38}$ at ca. $292 \mathrm{eV}$ but also oxygen-containing functional groups $(\mathrm{O}-\mathrm{C}=\mathrm{O}, \mathrm{C}=\mathrm{O}, \mathrm{C}-\mathrm{O})^{34,35}$ at ca. $289-286 \mathrm{eV}$ and carbon groups $(\mathrm{C}-\mathrm{C}, \mathrm{C}=\mathrm{C})^{39}$ at ca. $286-284 \mathrm{eV}$, as shown in Fig. 2a. Additionally, the as-received PTFE sample exhibited no peaks, whereas the HAP-treated PTFE sample exhibited a broad peak at ca. 531-537 eV, as shown in Fig. 2 b. These results indicated that the PTFE surface was oxidized upon HAP treatment. Furthermore, peak resolution was conducted for C1s-XPS spectra of as-received and HAP-treated PTFE samples. Figure 2c, $2 \mathrm{~d}$ shows peak resolution of the C1s-XPS spectra of the PTFE samples before and after HAP treatment. Peaks 1-8 indicate $\mathrm{CF}_{3}, \mathrm{CF}_{2}$, $\mathrm{C}-\mathrm{F}, \mathrm{O}-\mathrm{C}=\mathrm{O}, \mathrm{C}=\mathrm{O}, \mathrm{C}-\mathrm{O}, \mathrm{C}-\mathrm{C}$, and $\mathrm{C}=\mathrm{C}$, respectively. Table 1 shows the functional group ratios on the PTFE 

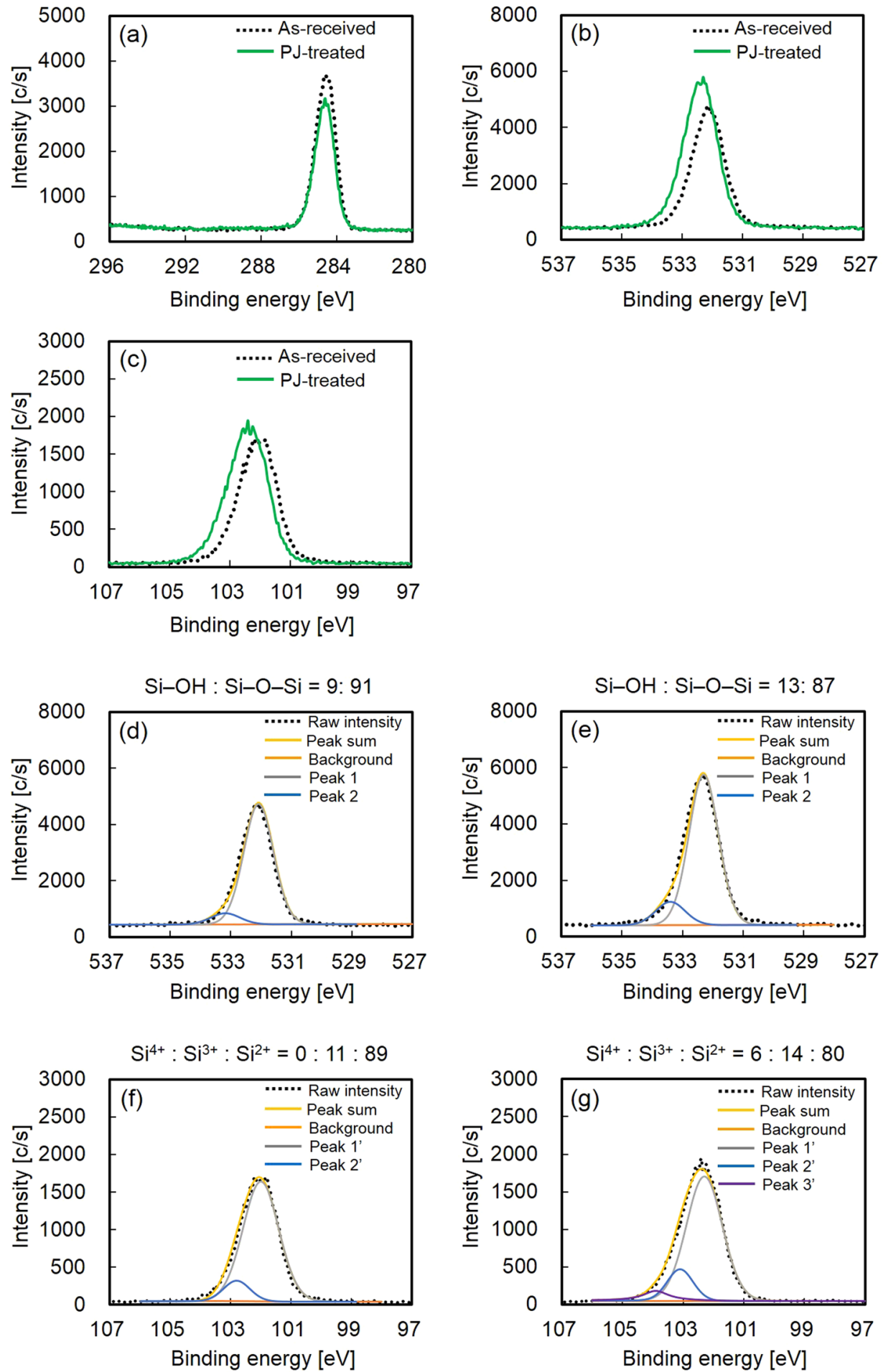

Figure 1. XPS spectra of the PDMS samples: (a) C1s-XPS spectra of the PDMS samples before and after PJ treatment, (b) O1s-XPS spectra of the PDMS samples before and after PJ treatment, (c) Si2p-XPS spectra of the PDMS samples before and after PJ treatment, (d) peak resolution of O1s-XPS spectrum of the PDMS sample before PJ treatment, (e) peak resolution of O1s-XPS spectrum of the PDMS sample after PJ treatment, (f) peak resolution of Si2p-XPS spectrum of the PDMS sample before PJ treatment, and ( $g$ ) peak resolution of Si2pXPS spectrum of the PDMS sample after PJ treatment. Peaks 1 and 2 indicate $\mathrm{Si}-\mathrm{O}-\mathrm{Si}$ and $\mathrm{Si}-\mathrm{OH}$, respectively. Peaks $1^{\prime}, 2^{\prime}$ and $3^{\prime}$ indicate $\mathrm{Si}^{2+}:-\left[\mathrm{Si}\left(\mathrm{CH}_{3}\right)_{2}-\mathrm{O}-\right]-, \mathrm{Si}^{3+}:-\left[\mathrm{Si}\left(\mathrm{CH}_{3}\right)(\mathrm{OH})-\mathrm{O}-\right]-$, and $\mathrm{Si}^{4+}:-\left[\mathrm{Si}(\mathrm{OH})_{2}-\mathrm{O}_{-}\right]-$, respectively. 

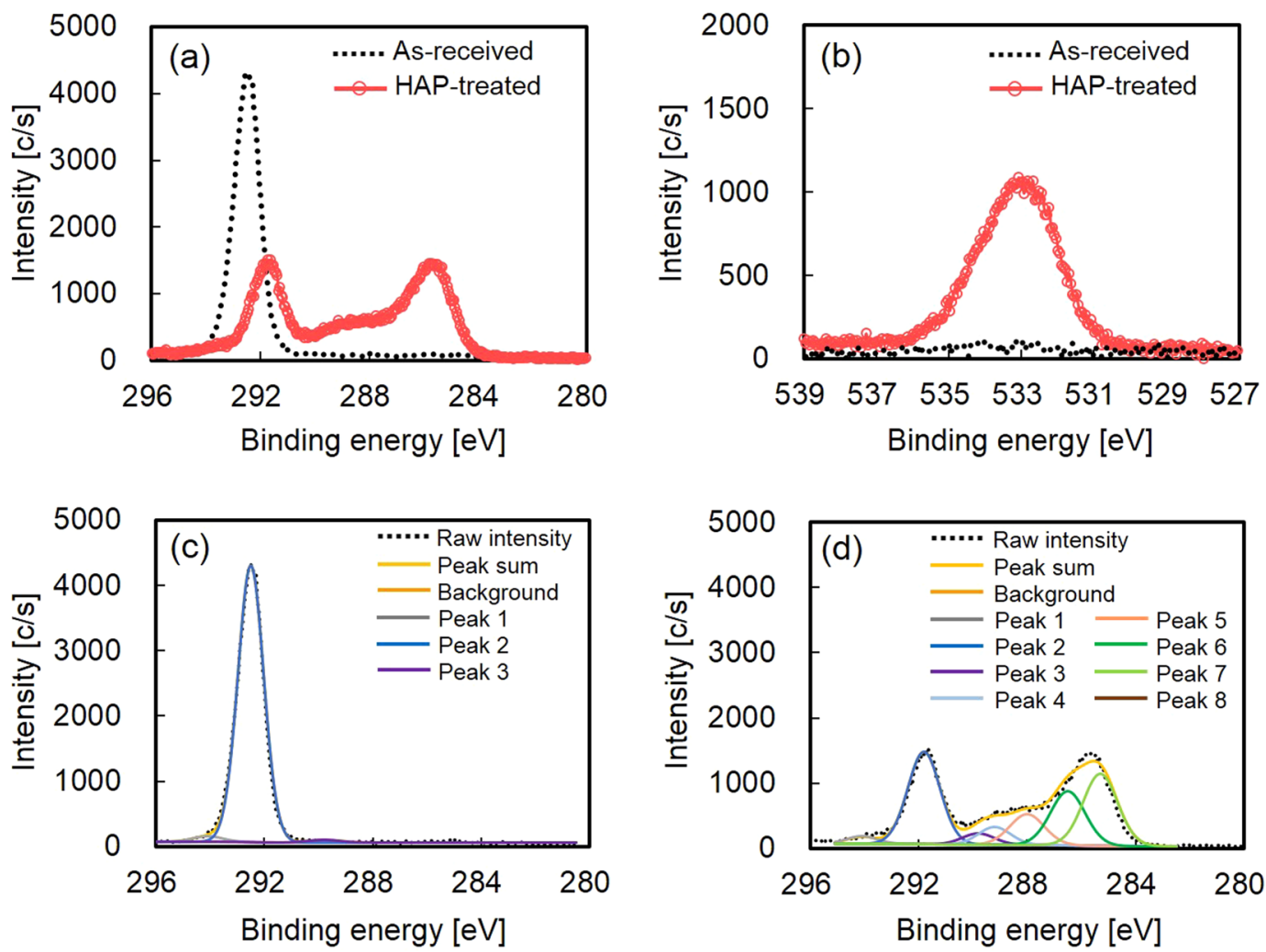

Figure 2. XPS spectra of the PTFE samples: (a) C1s-XPS spectra of the PTFE samples before and after HAP treatment, (b) O1s-XPS spectra of the PTFE samples before and after HAP treatment, (c) peak resolution of C1s-XPS spectrum of the PTFE sample before HAP treatment, and (d) peak resolution of C1s-XPS spectrum of the PTFE sample after HAP treatment. Peaks 1-8 indicate $\mathrm{CF}_{3}, \mathrm{CF}_{2}, \mathrm{C}-\mathrm{F}, \mathrm{O}-\mathrm{C}=\mathrm{O}, \mathrm{C}=\mathrm{O}, \mathrm{C}-\mathrm{O}, \mathrm{C}-\mathrm{C}$, and $\mathrm{C}=\mathrm{C}$, respectively.

\begin{tabular}{|l|l|l|l|l|}
\hline & & & \multirow{2}{*}{ As-received } & Unit [\%] \\
\cline { 5 - 5 } Peak No. & \multirow{2}{*}{ Functional group } & HAP treatment \\
\hline Peak 1 & $\mathrm{CF}_{3}$ & $294.1 \mathrm{eV}$ & 2.3 & 2.2 \\
\hline Peak 2 & $\mathrm{CF}_{2}$ & $\begin{array}{l}292.5^{*} \text { or } \\
291.8^{* *} \mathrm{eV}\end{array}$ & $96.6^{*}$ & $31.3^{* *}$ \\
\hline Peak 3 & $\mathrm{C}-\mathrm{F}$ & $289.8 \mathrm{eV}$ & 1.1 & 3.7 \\
\hline Peak 4 & $\mathrm{O}-\mathrm{C}=\mathrm{O}$ & $289.2 \mathrm{eV}$ & 0.0 & 5.9 \\
\hline Peak 5 & $\mathrm{C}=\mathrm{O}$ & $288.0 \mathrm{eV}$ & 0.0 & 11.0 \\
\hline Peak 6 & $\mathrm{C}-\mathrm{O}$ & $286.5 \mathrm{eV}$ & 0.0 & 19.7 \\
\hline Peak 7 & $\mathrm{C}-\mathrm{C}$ & $285.3 \mathrm{eV}$ & 0.0 & 26.2 \\
\hline Peak 8 & $\mathrm{C}=\mathrm{C}$ & $284.3 \mathrm{eV}$ & 0.0 & 0.0 \\
\hline
\end{tabular}

Table 1. Ratios of functional groups on the PTFE samples before and after HAP treatment, calculated from C1s-XPS spectra shown in Fig. 2c,2d. * and ** indicate that the obtained XPS spectra of as-received and HAPtreated PTFE samples were referenced to peaks indexed to $-\mathrm{CF}_{2}-$ at $292.5 \mathrm{eV}^{34,35}$ and $291.8 \mathrm{eV}^{13,38}$, respectively.

samples before and after HAP treatment, calculated from the C1s-XPS spectra shown in Fig. 2c,2d. These results indicated that both $\mathrm{C}-\mathrm{C}$ crosslinking and the generation of oxygen-containing functional groups occurred on the HAP-treated PTFE surface.

Adhesion strength between plasma-treated fluoropolymers and PDMS. Table 2 shows the sample preparation conditions and adhesion strengths of the PTFE/PDMS assembly. When the as-received PDMS or PTFE samples were used, the adhesion strength of the PTFE/PDMS assembly was $0 \mathrm{~N} / \mathrm{mm}$. Thus, the vulcanized PDMS sample required PJ treatment to strongly adhere to plasma-treated PTFE, although unvulcanized natural rubber (NR) containing hydrophilic $\mathrm{SiO}_{2}$ powder did not require plasma treatment. When both sides of PDMS and PTFE were plasma-treated, the adhesion strength of the PTFE/PDMS assembly was $2.6 \mathrm{~N} / \mathrm{mm}$, which indicated drastic increase of adhesion strength. The load-displacement curve of the PTFE/PDMS assembly was shown in Supplementary Information Fig. S1(a). Figure 3 shows the photographs of the PTFE/PDMS assembly during 


\begin{tabular}{|l|l|l|}
\hline \multicolumn{2}{|l|}{ Sample condition } & \multirow{2}{*}{ Adhesion strength $[\mathrm{N} / \mathrm{mm}]$} \\
\hline PDMS & PTFE & $0.0 \pm 0.0$ \\
\hline$\times$ & $\times$ & $0.0 \pm 0.0$ \\
\hline$\times$ & $O^{* *}$ & $0.0 \pm 0.0$ \\
\hline O* $^{*}$ & $\times$ & $2.6 \pm 0.2^{* * *}$ \\
\hline
\end{tabular}

Table 2. Sample preparation conditions and the adhesion strengths of PTFE/PDMS assembly. " $\times$ ” denotes no

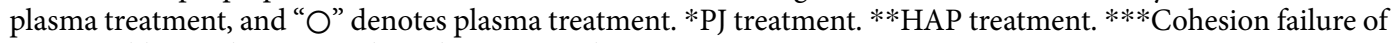
PDMS rubber and/or PTFE sheet during T-peel test.
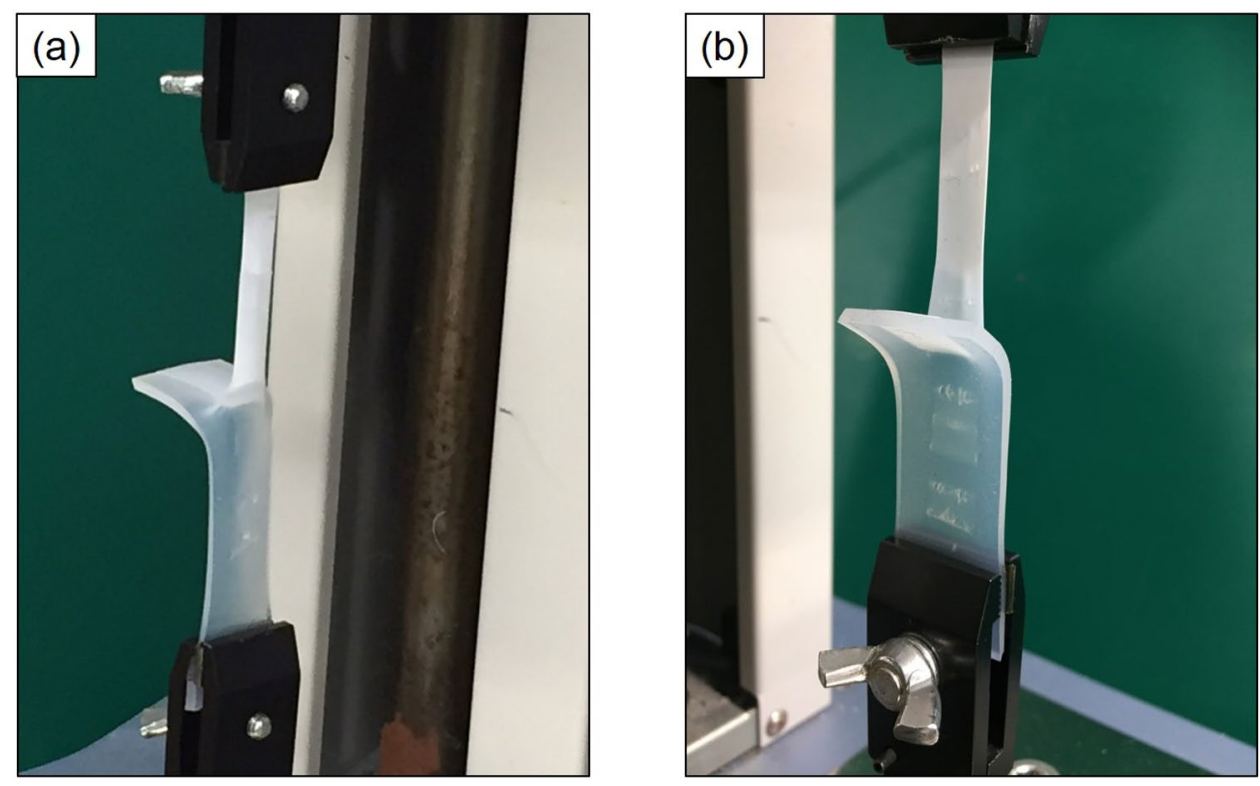

Figure 3. Photographs of PTFE/PDMS assembly during the T-peel test: (a) front side and (b) back side.

the T-peel test, where both sides of the PDMS and PTFE sheets were plasma-treated. Both the PDMS and PTFE sheets were extended during the T-peel test (Fig. 3a). Figure $3 \mathrm{~b}$ shows that PDMS which was strongly adhered to the PTFE surface was transferred to the PTFE side after the peel test. Figure 4 shows the XPS spectra and schematic of the peeled surfaces of the PTFE/PDMS assembly sample. Si was detected on both the PTFE and PDMS sides (Fig. $4 \mathrm{a}, 4 \mathrm{~b}$ ). $\mathrm{C}-\mathrm{H}$ but not $\mathrm{CF}_{2}$ was detected on the PDMS side, while the peak indexed to $\mathrm{C}-\mathrm{H}$ had a higher intensity than that indexed to $\mathrm{CF}_{2}$ on the PTFE side (Fig. 4c,4d). Thus, most of the PTFE surface was covered with PDMS after the peel test and a small amount of $\mathrm{CF}_{2}$ was detected at $292.5 \mathrm{eV}$ but not at $291.8 \mathrm{eV}$, because the PTFE sheet was extended during the T-peel test because of strong adhesion of the interface between PTFE and PDMS, after which bulk PTFE appeared on the surface, as illustrated in Fig. 4g. These results indicated cohesion failure of both PDMS and PTFE occurred when HAP-treated PTFE adhered strongly to PJ-treated PDMS. In summary, the adhesion strength of PTFE/PDMS interface was higher than breaking strengths of both PDMS and PTFE. For comparison, when PTFE was plasma-treated at low temperature (below $100^{\circ} \mathrm{C}$ ), the PTFE was thermally compressed to PJ-treated PDMS, and the PTFE/PDMS adhesion strength was $0.1 \mathrm{~N} / \mathrm{mm}$, which was extremely low. The effect of heating during plasma treatment was also confirmed during this study. HAP treatment was essential for obtaining high adhesion strength in the PTFE/PDMS assembly.

Rather than PTFE, tetrafluoroethylene-perfluoroalkylvinylether copolymer (PFA) sheet was HAP-treated at $19.1 \mathrm{~W} / \mathrm{cm}^{2}$ before preparing a PFA/PDMS assembly by thermal compression of the PJ-treated PDMS and HAP-treated PFA sheets. The adhesion strength of the PFA/PDMS assembly, in which the thicknesses of the PFA and PDMS sheets were 0.1 and $2 \mathrm{~mm}$, respectively, exceeded $2 \mathrm{~N} / \mathrm{mm}$, which indicated strong adhesive-free adhesion between the PDMS and PFA sheets. Figure 5 shows the photographs of PTFE/PDMS and PFA/PDMS assemblies on a paper showing the school badge of Osaka University. Although the badge was not observed under the PTFE/PDMS assembly, it was clearly observed under the PFA/PDMS assembly. The optical transparency of the PFA/PDMS assembly would allow observation of liquid flow in a PFA/PDMS assembly hose and/or tube, which would have high flexibility, chemical resistance, and weather resistance.

Strong adhesive-free adhesion between PTFE and other types of materials (metal and glass) via PJ-treated PDMS. As described in the Introduction section, it was previously reported that plasma-treated PDMS could strongly adhere to several materials such as metal and glass ${ }^{27-30}$. We attempted adhesive-free 

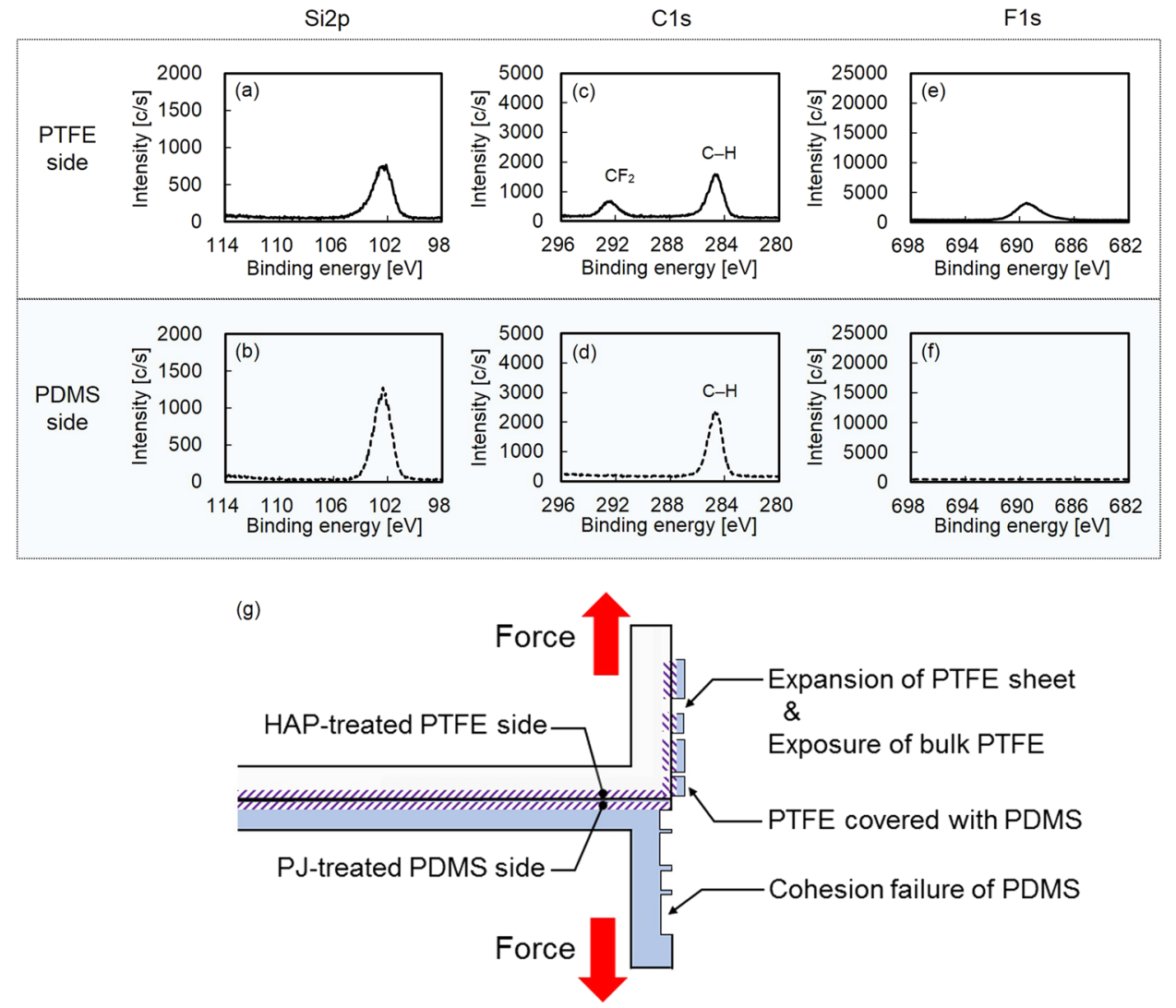

Figure 4. XPS spectra of the peeled surfaces of the PTFE/PDMS assembly sample in which both sides of PDMS and PTFE sheets were plasma-treated: (a) Si2p-XPS spectrum of the PTFE side, (b) Si2p-XPS spectrum of the PDMS side, (c) C1s-XPS spectrum of the PTFE side, (d) C1s-XPS spectrum of the PDMS side, (e) F1s-XPS spectrum of the PTFE side, (f) F1s-XPS spectrum of the PDMS side, and (g) schematic of peeled surfaces.

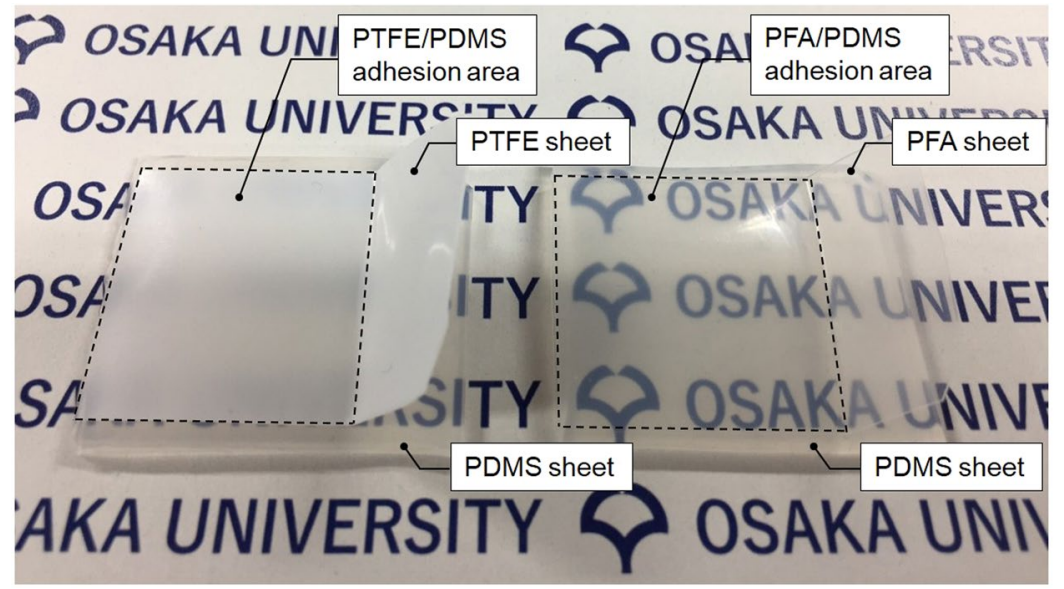

Figure 5. Photographs of PTFE/PDMS and PFA/PDMS assemblies on a paper showing the school badge of Osaka University. The thicknesses of the PTFE, PFA, and PDMS sheets were $0.2,0.1$ and $2 \mathrm{~mm}$, respectively.

adhesion between fluoropolymers (PTFE, PFA) and other types of materials such as metal and glass by combining of a technique reported previously with that developed in this study. We confirmed whether PJ-treated PDMS readily adhered to metal (copper and stainless steel) or glass. The load-displacement curve of the assemblies of Cu/ PDMS, SUS430/PDMS, and PDMS/glass were shown in Supplementary Information Fig. S1(b)-(d). The photographs of Cu/PDMS, SUS430/PDMS, and PDMS/glass were shown in Supplementary Information Fig. S2(a)-(c). We then prepared three-layer assemblies: PTFE/PDMS/metal and PTFE/PDMS/glass, as shown in Fig. 6. It was shown that strong adhesive-free adhesion between fluoropolymers (PTFE, PFA) and other types of materials such as metal and glass was readily realized by PJ treatment of both sides of a PDMS sheet. 
(a)
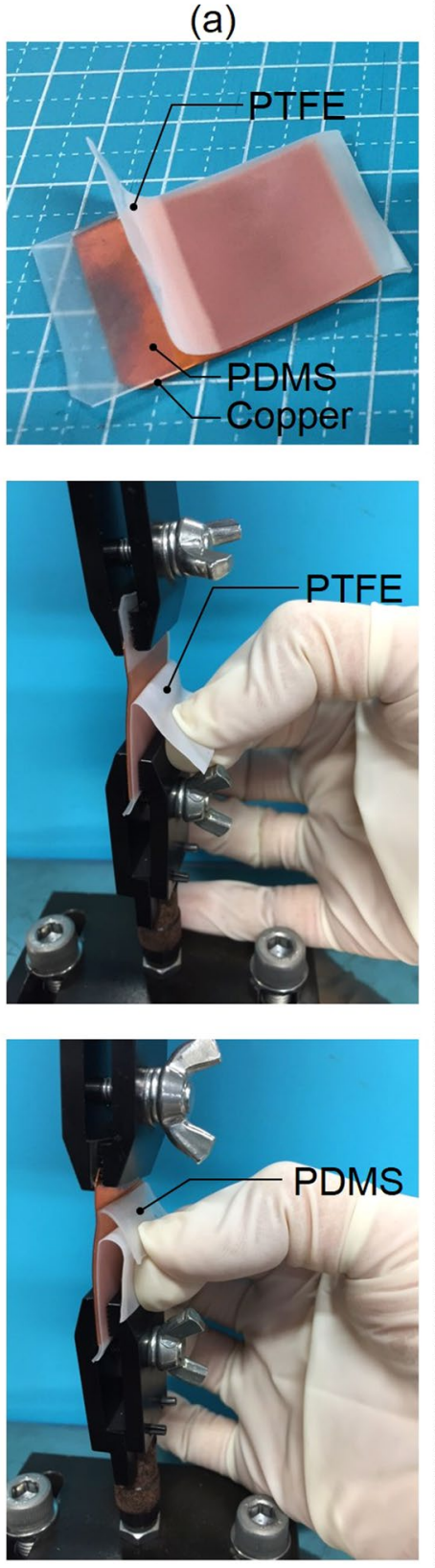

(b)
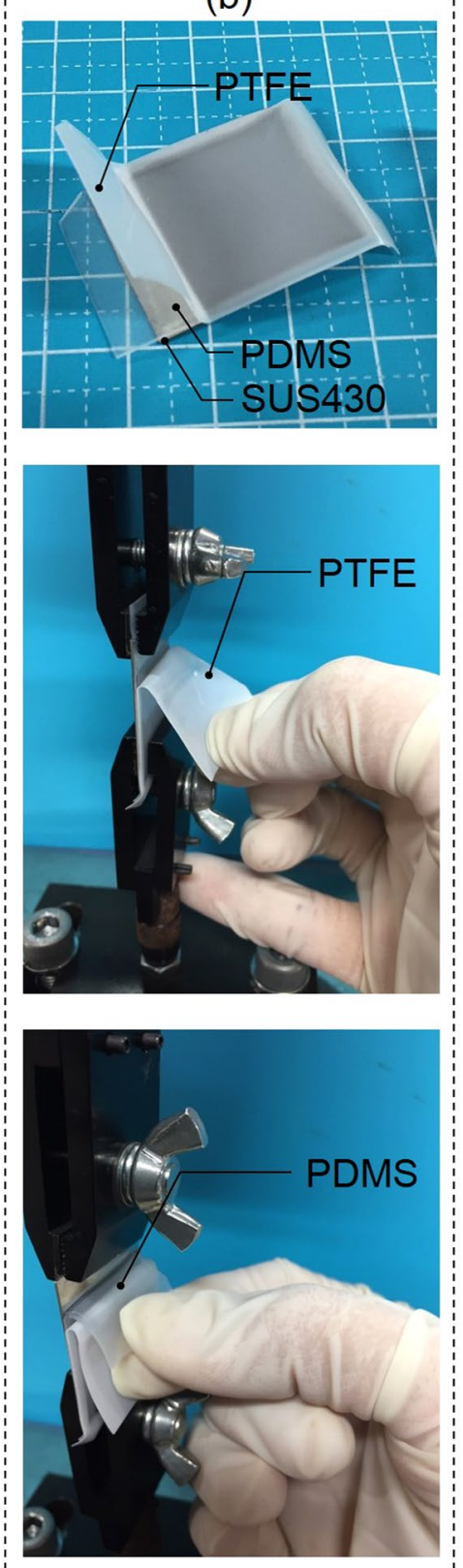

(c)
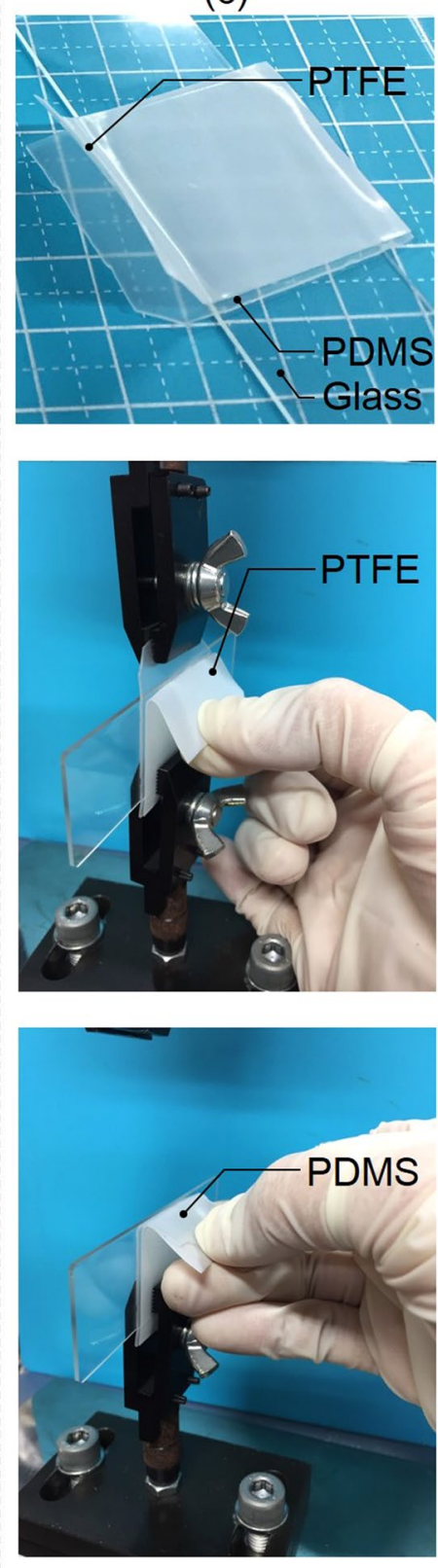

Figure 6. Photograph of three-layer assemblies of (a) PTFE/PDMS/Cu, (b) PTFE/PDMS/SUS430, and (c) PTFE/PDMS/glass. When the PTFE or PDMS sheet was jerked and shaken, no peeling occurred at the interfaces of PTFE/PDMS, PDMS/Cu, PDMS/SUS430, and PDMS/glass. It was shown that PJ-treated PDMS could be used as an alternative to strong adhesives to stick fluoropolymers to other types of materials. The adhesion strengths of the interfaces of PTFE/PDMS, PDMS/Cu, PDMS/SUS430, and PDMS/glass were measured using a $90^{\circ}$ peel test, and all the adhesion strengths exceeded $2 \mathrm{~N} / \mathrm{mm}$.

\section{Discussion}

In this study, vulcanized PDMS rubber was employed as an adherent for fluoropolymers, rather than unvulcanized rubber, which contains hydrophilic $\mathrm{SiO}_{2}$ powder containing $\mathrm{Si}-\mathrm{OH}$ groups. Although unvulcanized rubber adhered strongly to HAP-treated fluoropolymers without PJ treatment of the rubber in a previous study ${ }^{14}$, vulcanized rubber did not adhere to HAP-treated fluoropolymers without PJ treatment in the present study. When a vulcanized PDMS rubber was subjected to PJ treatment, we realized strong adhesive-free adhesion between the HAP-treated fluoropolymer and vulcanized PDMS. It was clear that this was achieved by plasma treatment of both sides of the fluoropolymer and vulcanized rubber.

Figure 7 shows a proposed model for strong adhesive-free adhesion as well as a preparation procedure for a two-layer assembly such as PTFE/PDMS and a three-layer assembly such as PTFE/PDMS/Cu. Firstly, a PTFE sheet is HAP-treated above $200^{\circ} \mathrm{C}$; oxygen-containing functional groups $(\mathrm{C}(=\mathrm{O})-\mathrm{OH}, \mathrm{C}-\mathrm{OH})$ are then generated and surface hardening occurs on the PTFE surface via HAP treatment. Secondly, a PDMS sheet is PJ-treated, 
Step 1

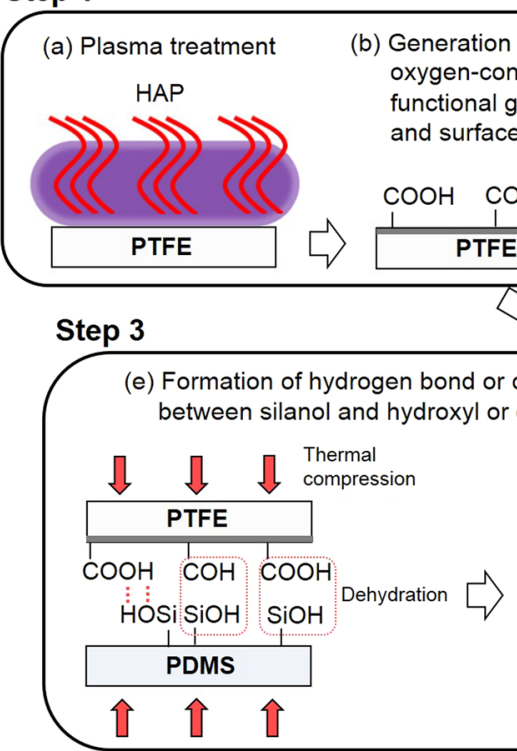

Step 2

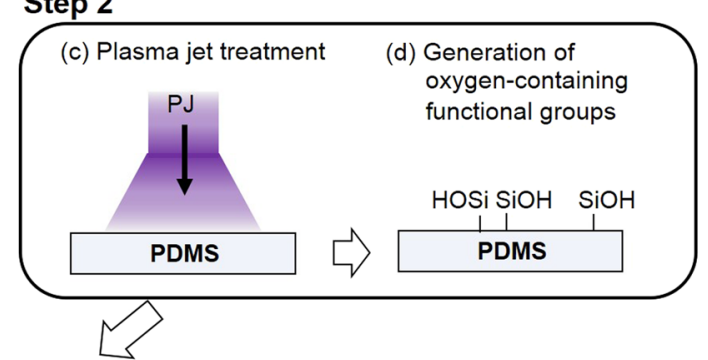

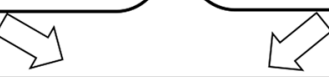

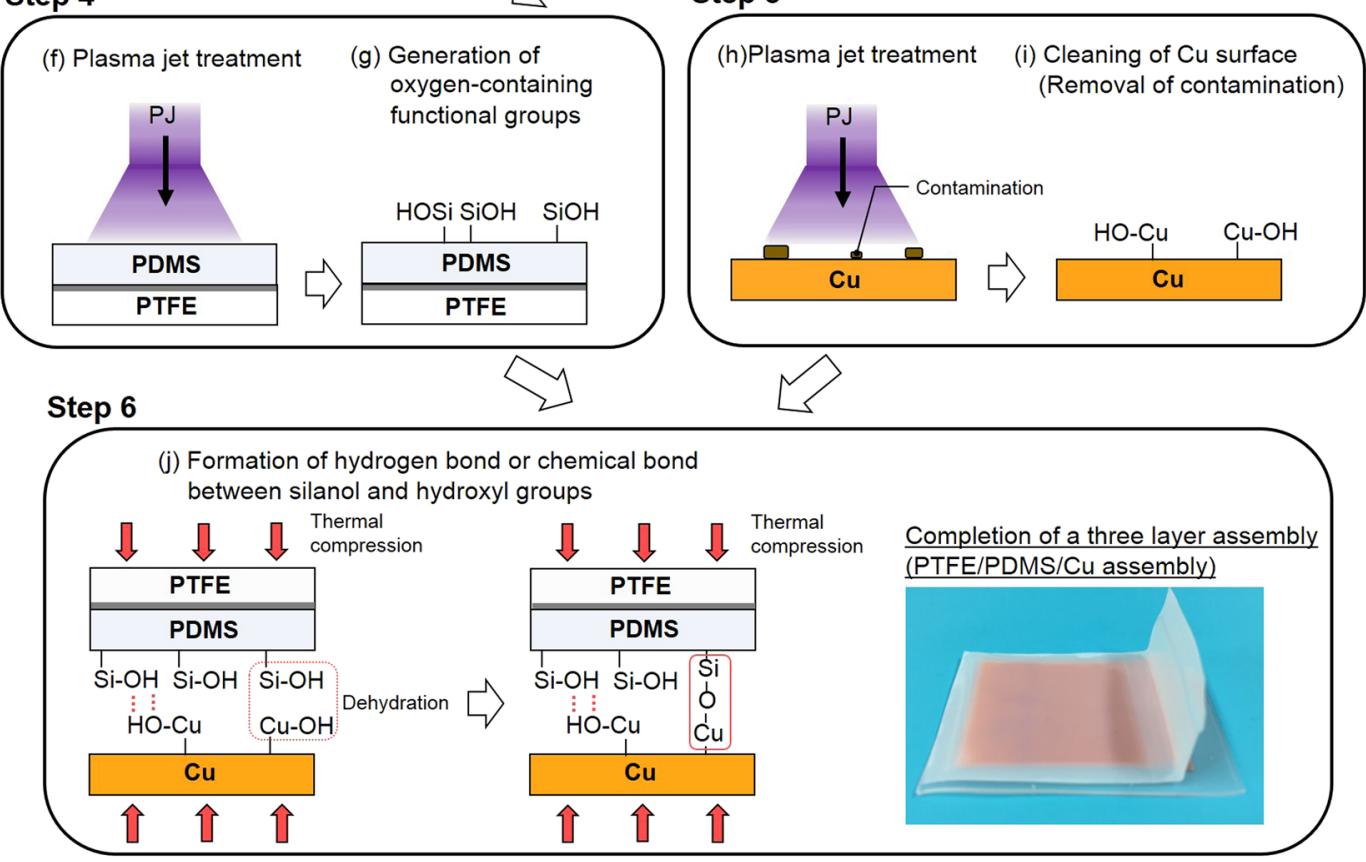

Figure 7. Proposed model for strong adhesion and preparation procedure for a two-layer assembly such as PTFE/PDMS (steps 1-3) and a three-layer assembly such as PTFE/PDMS/Cu (steps 1-6). PFA/PDMS, PFA/ PDMS/glass, PTFE/PDMS/glass, and PTFE/PDMS/SUS430 assemblies were prepared in the same way.

and silanol groups ( $\mathrm{Si}-\mathrm{OH}$ ) are generated on the PDMS surface. Thirdly, thermal compression of the HAP-treated PTFE sheet and the PJ-treated PDMS sheet is conducted, forming hydrogen bonds between silanol groups on the PJ-treated PDMS surface and hydroxyl or carboxyl groups on the HAP-treated PTFE surface. Additionally, dehydration condensation occurs between silanol and hydroxyl or carboxyl groups, forming $\mathrm{C}-\mathrm{O}-\mathrm{Si}$ and/or $\mathrm{C}(=\mathrm{O})-\mathrm{O}-\mathrm{Si}$ bonds. Considering that cohesion failure of both PDMS and PTFE occurred when PJ-treated PDMS and HAP-treated PTFE were thermally compressed, it is reasonable to assume that not only hydrogen bonds but also covalent bonds $(\mathrm{C}-\mathrm{O}-\mathrm{Si}$ and/or $\mathrm{C}(=\mathrm{O})-\mathrm{O}-\mathrm{Si})$ were formed. Fourthly, the side of the PDMS surface of the PTFE/PDMS assembly is PJ-treated, generating silanol groups ( $\mathrm{Si}-\mathrm{OH})$ on the PDMS surface. Fifthly, a copper plate is also PJ-treated to clean its surface; contamination is then removed, and hydroxyl groups $(\mathrm{Cu}-\mathrm{OH})$ are generated on the $\mathrm{Cu}$ surface. Finally, thermal compression of the PJ-treated PDMS/PTFE assembly and the PJ-treated $\mathrm{Cu}$ plate is performed, resulting in formation of hydrogen bonds between silanol groups on the PJ-treated PDMS/PTFE surface and hydroxyl groups on the PJ-treated Cu surface. Additionally, dehydration condensation occurs between silanol and hydroxyl groups, resulting in $\mathrm{Si}-\mathrm{O}-\mathrm{Cu}$ bond formation. Considering 
that cohesion failure of PDMS occurred when PJ-treated PDMS and PJ-treated Cu were thermally compressed, it is reasonable to assume that not only hydrogen bonds but also covalent bonds $(\mathrm{Si}-\mathrm{O}-\mathrm{Cu})$ are formed.

This technique for achieving strong adhesive-free adhesion between PDMS and fluoropolymer adds several functions, such as high chemical resistance, water and oil repellency, antifouling and sliding properties, to PDMS, and elasticity to fluoropolymers. Additionally, three-layer assemblies such as PTFE/PDMS/metal and PTFE/ PDMS/glass were successfully prepared by PJ treatment of both sides of a PDMS sheet. This demonstrated that PJ-treated PDMS can adhere fluoropolymers to several materials such as metal and glass as desired. In summary, this technique for achieving adhesive-free adhesion between PDMS combined with fluoropolymer will expand the application range of PDMS and fluoropolymers will be useful in various fields, especially the medical and food industries, in which adhesives are unsuitable.

\begin{abstract}
Methods
Materials. An addition-crosslinked PDMS sheet (KE541U + C25A/B, HATADA, hardness $40^{\circ}$ ) was cut into $35 \mathrm{~mm} \times 50 \mathrm{~mm}$ pieces, which was used as a silicone rubber specimen. A PDMS sheet with a thickness of $2 \mathrm{~mm}$ was mainly used, but a PDMS sheet with a thickness of $0.5 \mathrm{~mm}$ was also used for realization at high optical transparency. Commercially available PTFE sheet (NITOFLON ${ }^{\circledR}$ No. 900UL, Nitto Denko; thickness: $0.2 \mathrm{~mm}$ ) was cut into $45 \mathrm{~mm} \times 70 \mathrm{~mm}$ pieces, which was used as a fluoropolymer specimen. The color of PTFE sheet is white and PTFE has no optical transparency due to its high crystallinity. Conversely, the PFA sheet originally has optical transparency as well as PDMS sheet. Therefore, the PFA sheet (AF-0100, DAIKIN INDUSTRIES; thickness: $0.1 \mathrm{~mm}$ ) was used only when high transparency was required. Copper foil (Cu, 99.9\%, CU-113263, Nilaco Corporation; thickness: $0.050 \mathrm{~mm}$ ) was cut into $30 \mathrm{~mm} \times 25 \mathrm{~mm}$ pieces, which was used as a metal foil specimen. A pure copper plate (Cu, 99.96\%, CU-113381, Nilaco Corporation; thickness: $0.20 \mathrm{~mm})$ was also cut into $30 \mathrm{~mm} \times 25 \mathrm{~mm}$ pieces, which was used as a Cu plate specimen. Stainless steel foil (SUS304, TS200-200-005, IWATA MFG; thickness: $0.05 \mathrm{~mm}$ ) was cut into $30 \mathrm{~mm} \times 25 \mathrm{~mm}$ pieces, which was used as a SUS foil specimen. A stainless steel plate (SUS430, HSO531, Hikari; thickness: $0.5 \mathrm{~mm}$ ) was also cut into $30 \mathrm{~mm} \times 25 \mathrm{~mm}$ pieces, which was used as a SUS plate specimen. A glass slide (S7213, Matsunami Glass Ind.) with $76 \mathrm{~mm} \times 26 \mathrm{~mm} \times 1 \mathrm{~mm}$ was used without cutting.
\end{abstract}

Sample preparation by plasma treatment. Prior to PJ treatment, the PDMS sheets were washed sequentially with acetone $(99.5 \%$, Kishida Chemical) and pure water for $1 \mathrm{~min}$ each in an ultrasonic bath (US-4R, AS- ONE). The washed PDMS sheets were then dried using an air gun containing $\mathrm{N}_{2}$ gas (99.99\%, Iwatani Fine Gas). The washed and dried PDMS sheets were then plasma-treated using open-air-type PJ treatment equipment (Tough Plasma FPE-20, FUJI CORPORATION), but not HAP-treated. The gap between the irradiation hole and the surface of the PDMS sheet was $10 \mathrm{~mm}$; a mixture of $\mathrm{N}_{2}$ gas $\left(99.99 \%\right.$, Iwatani Fine Gas) and air gas $\left(\mathrm{N}_{2} /\right.$ $\mathrm{O}_{2}=79 \% / 21 \%$, Iwatani Fine Gas) was used as a process gas for plasma generation, and the flow rates of $\mathrm{N}_{2}$ and air gases were 29.7 and $0.3 \mathrm{~L} / \mathrm{min}$, respectively. The PDMS sheet was placed on a movable stage and fixed using double-sided polyimide tape (10-mm width, No. 4390, $3 \mathrm{M}$ Japan), then the stage was moved at $8 \mathrm{~mm} / \mathrm{s}$ during $\mathrm{PJ}$ treatment. The number of scan operations was only one in this study. To confirm whether the PJ treatment conditions were suitable, two PJ-treated PDMS sheets were prepared under the same PJ treatment conditions; the adhesion strength of the PDMS/PDMS assembly was then measured using a T-peel test. Cohesion failure of the PDMS occurred during the T-peel test, as shown in Supplementary Information Fig. S2(d). It was confirmed that these PJ conditions were suitable for PDMS.

It was previously reported that low-temperature plasma treatment barely improved the adhesion properties of PTFE, whereas HAP treatment significant improved them ${ }^{12,13}$. On the basis of these reports, the surface of the PTFE sheet was modified via HAP treatment in the present study. The detailed surface treatment procedure for a PTFE sheet using HAP has been previously reported ${ }^{13}$; hence, it is described only briefly here. Prior to HAP treatment, PTFE sheets as well as PDMS sheets were washed with acetone and pure water using an ultrasonic bath (US-4R, AS- ONE) then dried using an air gun containing $\mathrm{N}_{2}$ gas (99.99\%, Iwatani Fine Gas). The washed and dried PTFE sheets were then HAP-treated at $19.1 \mathrm{~W} / \mathrm{cm}^{2}$ for $600 \mathrm{~s}$ using He gas (99.99\%, Iwatani Fine Gas) at atmospheric pressure in a custom-made chamber system (Meisyo Kiko) ${ }^{12-14}$. During HAP treatment, the surface temperature of the PTFE samples was measured with a digital radiation thermometer system (FT-H40K and FT-50A, Keyence); it was confirmed that the maximum surface temperature exceeded $200^{\circ} \mathrm{C}$ during HAP treatment at $19.1 \mathrm{~W} / \mathrm{cm}^{2}$ for $600 \mathrm{~s}$. To confirm whether the HAP treatment conditions were suitable, an HAP-treated PTFE sheet and unvulcanized NR containing hydrophilic $\mathrm{SiO}_{2}$ powder were prepared; the adhesion strength of the PTFE/NR assembly was then measured using a T-peel test. Cohesion failure of the NR occurred during the T-peel test, as shown in Supplementary Information Fig. S2(e). It was thus confirmed that these HAP conditions were suitable for PTFE ${ }^{14}$

A glass slide was used without washing or PJ treatment. Cu foils, Cu plate, SUS foils, and SUS plates as well as PDMS sheets were washed in an ultrasonic bath (US-4R, AS- ONE) then dried using an air gun containing $\mathrm{N}_{2}$ gas (99.99\%, Iwatani Fine Gas). The washed and dried $\mathrm{Cu}$ foils, $\mathrm{Cu}$ plate, SUS foils, and SUS plates were also PJ-treated to clean their surfaces. The gap between the irradiation hole and the surface of the Cu foils, $\mathrm{Cu}$ plate, SUS foils, or SUS plates was $10 \mathrm{~mm}$. The stage was moved at $0.8 \mathrm{~mm} / \mathrm{s}$ during PJ treatment for Cu foils, Cu plate, SUS foils, or SUS plates. The number of scan operations was five for $\mathrm{Cu}$ foils, $\mathrm{Cu}$ plate, SUS foils, and SUS plates.

Surface chemical composition analysis. XPS measurements were performed using a scanning XPS

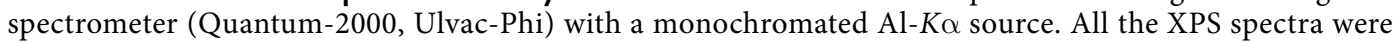
obtained below $5 \times 10^{-6} \mathrm{~Pa}$. The photoelectron take-off angle was $45^{\circ}$, and the $\mathrm{X}$-ray irradiation area was $\varnothing 100 \mu \mathrm{m}$. Narrow scan XPS spectra of Si2p, C1s, O1s, and F1s were collected at 95-115eV, 275-300 eV, 525$545 \mathrm{eV}$, and $680-700 \mathrm{eV}$, respectively, with a pass energy of $23.50 \mathrm{eV}$ and a step size of $0.05 \mathrm{eV}$. The cumulative 
number of measurements was three. During an XPS measurement, the samples were irradiated with a low-speed electron beam and an Ar ion beam to achieve charge neutralization. The obtained XPS spectra of the PDMS samples were referenced to the peak indexed to C-Si-O-Si and/or C-H at $284.6 \mathrm{eV}^{36,37}$, and the obtained XPS spectra of as-received and HAP-treated PTFE samples were referenced to peaks indexed to $-\mathrm{CF}_{2}-$ at $292.5 \mathrm{eV}^{34,35}$ and $291.8 \mathrm{eV}^{13,38}$, respectively. The main peak of $-\mathrm{CF}_{2}-$ in the $\mathrm{C} 1 \mathrm{~s}$-XPS spectra shifted toward lower binding energy for plasma-treated PTFE due to surface charging ${ }^{13,40}$.

Adhesion strength test for a two-layer assembly. Firstly, the HAP-treated PTFE sample was placed on the PJ-treated PDMS sheets in a mold such that the plasma-treated surfaces faced each other. Secondly, the $\mathrm{PTFE} / \mathrm{PDMS}$ assembly was compressed without adhesive at $180^{\circ} \mathrm{C}$ and $10 \mathrm{MPa}$ for $10 \mathrm{~min}$ using a hot-pressing machine (AH-2003, AS-ONE). Thirdly, the PTFE/PDMS assembly was restored to room temperature. Fourthly, the adhesion strength of the PTFE/PDMS assembly was measured using a T-peel test by combining of a digital force gauge (ZP-200N, Imada) and an electric-driven stand (MX-500N, Imada). The T-peel test was conducted at room temperature of $25 \pm 5^{\circ} \mathrm{C}$, and the humidity was not controlled. The sweep rate was $60 \mathrm{~mm} / \mathrm{min}$. Finally, the average adhesion strength was calculated by dividing the average tensile strength by the width of the PTFE (ca. $10 \mathrm{~mm}$ ). To verify the reproducibility, three samples were prepared under the same conditions. A PFA/PDMS assembly was also prepared, and its adhesion strength was measured as for the PTFE/PDMS assembly.

Adhesion strength test for a three-layer assembly. The first to third steps for a three-layer assembly were the same as those for a two-layer assembly. Fourthly, a PJ-treated PDMS/PTFE sample was placed on a PJ-treated $\mathrm{Cu}$ plate in a mold so that the plasma-treated surfaces faced each other. Fifthly, the PTFE/PDMS/ $\mathrm{Cu}$ assembly was compressed without adhesive at $180^{\circ} \mathrm{C}$ and $5 \mathrm{MPa}$ for $10 \mathrm{~min}$ using a hot-pressing machine (AH-2003, AS-ONE). Sixthly, the PTFE/PDMS/Cu assembly was restored to room temperature. Seventhly, the PTFE and PDMS sheet on the Cu plate was cut to a width of $10 \mathrm{~mm}$. Eighthly, the Cu plate was fixed on the electric-driven stand (MX-500N, Imada) using two stainless steel bars, then both the PTFE and PDMS sheets were simultaneously grasped and pulled up when measuring the adhesion strength of the PDMS/Cu interface with a $90^{\circ}$ peel test. The $90^{\circ}$ peel test was conducted under the same conditions of temperature and humidity as T-peel test. The sweep rate was also $60 \mathrm{~mm} / \mathrm{min}$. When the adhesion strength of the PTFE/PDMS interface was measured using a $90^{\circ}$ peel test, the $\mathrm{Cu}$ plate was fixed on the electric-driven stand using two stainless steel bars, and then only the PTFE sheet was pulled up. The PTFE/PDMS/SUS430 assembly was prepared as for the $\mathrm{PTFE} / \mathrm{PDMS} / \mathrm{Cu}$ assembly. The PFA/PDMS/glass and PTFE/PDMS/glass assemblies were prepared as for the $\mathrm{PTFE} / \mathrm{PDMS} / \mathrm{Cu}$ assembly except for the fifth step, in which the pressure was decreased from 5 to almost $0 \mathrm{MPa}$ (the empty weight of the PFA/PDMS or PTFE/PDMS assembly) because the glass slide was easily broken. The adhesion strengths of PFA/PDMS/glass, PTFE/PDMS/glass, and PTFE/PDMS/SUS430 were measured as for the $\mathrm{PTFE} / \mathrm{PDMS} / \mathrm{Cu}$ assembly. Peeling tests were conducted at least two times for each three-layer assembly to verify the reproducibility.

\section{References}

1. Schonhorn, H. \& Hansen, R. H. Surface treatment of polymers for adhesive bonding. J. Appl. Polym. Sci. 11, 1461-1474 (1967).

2. Bikerman, J. J. Causes of poor adhesion: weak boundary layers. Ind. Eng. Chem. 59, 40-44 (1967).

3. Miller, M. L., Postal, R. H., Sawyer, P. N., Martin, J. G. \& Kaplit, M. J. Conditioning polytetrafluoroethylene surfaces for use in vascular prostheses. J. Appl. Polym. Sci. 14, 257-266 (1970)

4. Dwight, D. W. \& Riggs, W. M. Fluoropolymer surface studies. J. Colloid. Interface Sci. 47, 650-660 (1974).

5. Marchesi, J. T., Keith, H. D. \& Garton, A. Adhesion to sodium naphthalenide treated fluoropolymers. part iii. mechanism of adhesion. J. Adhesion 39, 185-205 (1992).

6. Inagaki, N., Tasaka, S. \& Kawai, H. Improved adhesion of poly(tetrafluoroethylene) by $\mathrm{NH}_{3}$-plasma treatment. J. Adhesion Sci. Tech. 3, 637-649 (1989).

7. Kogoma, M., Takahashi, K. \& Tanaka, K. Improvement of adhesive strength of polytetrafluoroethylene by defluorination treatment with combination of atmospheric pressure glow plasma treatment and chemical transport method. J. Photopolm. Sci. Tech. 3, 637-649 (2011)

8. Kang, E. T. \& Zhang, Y. Surface modification of fluoropolymers via molecular design. Adv. Mater. 12, 1481-1494 (2000).

9. Zhang, M. C., Kang, E. T., Neoh, K. G. \& Tan, K. L. Surface modification of aluminum foil and PTFE film by graft polymerization for adhesion enhancement. Colloid. Surf. A 176, 139-150 (2001).

10. Zhang, L., Chen, Y. \& Dong, T. Studies on the adhesion between polytetrafluoroethylene film and silanized glass foil. Surf. Interface Anal. 36, 311-316 (2004)

11. Okubo, M., Tahara, M., Aburatani, Y., Kuroki, T. \& Hibino, T. Preparation of PTFE film with adhesive surface treated by atmospheric-pressure nonthermal plasma graft polymerization. IEEE Trans. Ind. Appl. 46, 1715-1721 (2010).

12. Ohkubo, Y. et al. Adhesive-free adhesion between polytetrafluoroethylene (PTFE) and isobutylene-isoprene rubber (IIR) via heatassisted plasma treatment. RSC Adv. 7, 6432-6438 (2017).

13. Ohkubo, Y. et al. Drastic improvement in adhesion property of polytetrafluoroethylene (PTFE) via heat-assisted plasma treatment using a heater. Sci. Rep. 7 Art.no. 9476 (2017).

14. Ohkubo, Y. et al. Effect of rubber compounding agent on adhesion strength between rubber and heat-assisted plasma-treated polytetrafluoroethylene (PTFE). J. Adhesion; https://doi.org/10.1080/00218464.2018.1428095 (2018).

15. Hollahan, J. R. \& Carlson, G. L. Hydroxylation of polymethylsiloxane surfaces by oxidizing plasmas. J. Appl. Polym. Sci. 14, 2499-2508 (1970).

16. Efimenko, K., Wallace, W. E. \& Genzer, J. Surface modification of Sylgard-184 poly(dimethyl siloxane) networks by ultraviolet and ultraviolet/ozone treatment. J. Colloid. Interface Sci. 254, 306-315 (2002).

17. Haji, K., Zhu, Y., Otsubo, M. \& Honda, C. Surface modification of silicone rubber after corona exposure. Plasma Processes Polym. 4, S1075-S1080 (2007).

18. Anderson, J. R. et al. Fabrication of topologically complex three-dimensional microfluidic systems in PDMS by rapid prototyping. Anal. Chem. 72, 3158-3164 (2000).

19. McDonald, J. C. \& Whitesides, G. M. Poly(dimethylsiloxane) as a material for fabricating microfluidic devices. Accounts Chem. Res. 35, 491-499 (2002)

20. Fujii, T. PDMS-based microfluidic devices for biomedical applications. Microelectronic Eng. 61-62, 907-914 (2002) 
21. Zhou, J., Ellis, A. V. \& Voelcker, N. H. Recent developments in PDMS surface modification for microfluidic devices. Electrophoresis 31, 2-16 (2010).

22. Wu, H., Huang, B. \& Zare, R. N. Construction of microfluidic chips using polydimethylsiloxane for adhesive bonding. Lab on a Chip 5, 1393-1398 (2005).

23. Eddings, M. A., Johnson, M. A. \& Gale, B. K. Determining the optimal PDMS-PDMS bonding technique for microfluidic devices. J. Micromech. Microeng. 18, 067001 (2008).

24. De Geyter, N. et al. Remote atmospheric pressure DC glow discharge treatment for adhesion improvement of PDMS. Plasma Process. Polym. 6, S406-S411 (2009).

25. Vlachopoulou, M. E. et al. A low temperature surface modification assisted method for bonding plastic substrates. J. Micromech. Microeng. 19, 015007 (2009).

26. Sunkara, V. et al. Simple room temperature bonding of thermoplastics and poly(dimethylsiloxane). Lab on a Chip 11, 962-965 (2011).

27. Sunkara, V., Park, D. K. \& Cho, Y. K. Versatile method for bonding hard and soft materials. RSC Adv. 2, 9066-9070 (2012).

28. Gajasinghe, R. W. R. L. et al. Experimental study of PDMS bonding to various substrates for monolithic microfluidic applications. J. Micromech. Microeng. 24, 075010 (2014).

29. Bhattacharya, S., Datta, A., Berg, J. M. \& Gangopadhyay, S. Studies on surface wettability of poly(dimethyl) siloxane (PDMS) and glass under oxygen-plasma treatment and correlation with bond strength. J. Microelectromech. Systems 14, 590-597 (2005).

30. Samel, B., Chowdhury, M. K. \& Stemme, G. The fabrication of microfluidic structures by means of full-wafer adhesive bonding using a poly(dimethylsiloxane) catalyst. J. Micromech. Microeng. 17, 1710-1714 (2007).

31. Nishi, Y. et al. Effects of electron beam irradiation on adhesive force of laminated sheet of high strength polytetrafluoroethylene (PTFE) and bio-adaptable polydimethylsiloxane (PDMS). Mater. Trans. 53, 1657-1664 (2012).

32. Kubo, C., Yagi, A., Kanda, M. \& Nishi, Y. Effects of electron beam irradiation on shear strength of laminated sheet of bio-adaptable polydimethylsiloxane (PDMS) and polytetrafluoroethylene (PTFE) with fracture toughness. Mater. Trans. 56, 529-533 (2015).

33. Jin, X. et al. Joining the un-joinable: Adhesion between low surface energy polymers using tetrapodal ZnO linkers. Adv. Mater. 24, 5676-5680 (2012).

34. Vandencasteele, N. \& Reniers, F. Plasma-modified polymer surfaces: Characterization using XPS. J. Elec. Spec. Relat. Phenom. 178-179, 394-408 (2010).

35. Hubert, J. et al. Etching processes of polytetrafluoroethylene surfaces exposed to $\mathrm{He}$ and $\mathrm{He}-\mathrm{O}_{2}$ atmospheric post-discharges. Langmuir 28, 9466-9474 (2012).

36. Williams, R. L., Wilson, D. J. \& Rhodes, N. P. Stability of plasma-treated silicone rubber and its influence on the interfacial aspects of blood compatibility. Biomaterials 25, 4659-4673 (2004).

37. Satriano, C., Marietta, G. \& Kasemo, B. Oxygen plasma-induced conversion of polysiloxane into hydrophilic and smooth SiOx surfaces. Surf. Interface Anal. 40, 649-656 (2008).

38. Wilson, D. J., Williams, R. L. \& Pond, R. C. Plasma modification of PTFE surfaces. Part I: Surfaces immediately following plasma treatment. Surf. Interface Anal. 31, 385-396 (2001).

39. Díaz, J., Paolicelli, G., Ferrer, S. \& Comin, F. Separation of the $\mathrm{sp}^{3}$ and $\mathrm{sp}^{2}$ components in the $\mathrm{C} 1 \mathrm{~s}$ photoemission spectra of amorphous carbon films. Phys. Review B. 54, 8064-8069 (1996).

40. Momose, Y., Tamura, Y., Ogino, M., Okazaki, S. \& Hirayama. Chemical reactivity between Teflon surfaces subjected to argon plasma treatment and atmospheric oxygen. J. Vacuum Sci. Tech. 10, 229-238 (1992).

\section{Author Contributions}

Y.O., K.E. and K.Y. supervised the work. Y.O. performed plasma treatment for both the PTFE and PDMS samples. Y.O. also performed all the analysis. K.Y. and K.E. provided comments on the measurements. All authors contributed to the scientific discussion and manuscript preparation. Y.O. wrote the manuscript.

\section{Additional Information}

Supplementary information accompanies this paper at https://doi.org/10.1038/s41598-018-36469-y.

Competing Interests: The authors declare no competing interests.

Publisher's note: Springer Nature remains neutral with regard to jurisdictional claims in published maps and institutional affiliations.

(c) (1) Open Access This article is licensed under a Creative Commons Attribution 4.0 International

License, which permits use, sharing, adaptation, distribution and reproduction in any medium or format, as long as you give appropriate credit to the original author(s) and the source, provide a link to the Creative Commons license, and indicate if changes were made. The images or other third party material in this article are included in the article's Creative Commons license, unless indicated otherwise in a credit line to the material. If material is not included in the article's Creative Commons license and your intended use is not permitted by statutory regulation or exceeds the permitted use, you will need to obtain permission directly from the copyright holder. To view a copy of this license, visit http://creativecommons.org/licenses/by/4.0/.

(c) The Author(s) 2018 